\title{
Offenheit und Ausgewogenheit bei deontologischen Verfahren
}

\author{
Jean Martin \\ Dr. med., Mitglied der Redaktion
}

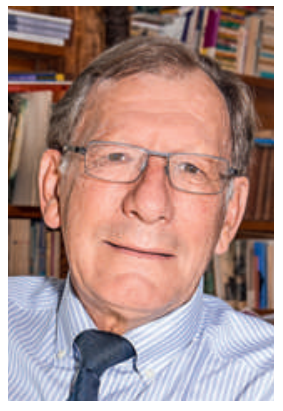

Wichtiger Beitrag in der ersten Ausgabe 2016 der SÄZ [1]: Die von S. Cueni und anderen beschriebenen Handlungen und Ausrichtungen der FMH sollten zu einer beträchtlichen Verbesserung der bestehenden Gepflogenheiten beitragen, die für die Patientinnen mit beträchtlichen Schwierigkeiten verbunden waren [2]. Ich erlaube mir, einige eigene Erfahrungen zu erwähnen: Als Kantonsarzt wandte ich mich in den 80er Jahren an meinen Kollegen im Nachbarkanton. Dabei ging es um einen in seinem Kanton praktizierenden Arzt, der seine Tätigkeit im Kanton Waadt ausüben wollte. Als Antwort erhielt ich aber den Hinweis, dass der Datenschutz einen Informationsaustausch nicht erlaube. Im Hinblick auf unseren Auftrag, die Gesundheit der Bevölkerung zu schützen, war diese Situation

Die von Cueni et al. beschriebenen Ausrichtungen sind notwendig und angebracht.

zumindest ärgerlich. Sollten wir - falls unser Kollege früher aus irgendeinem Grund unangenehm aufgefallen war - darauf warten, dass er bei uns erneut unangenehm auffallen würde? Eine weitere heikle Situation: Aus der Presse erfuhren wir, dass eine Gesundheitsfachperson wegen Beihilfe zu einem Mord in einem anderen Kanton verurteilt worden war. Der Betreffende focht unsere Entscheidung bezüglich einer Bewilligung für den Kanton Waadt jedoch an. Solche Fälle zeigen, wie sehr in einer immer mobiler werdenden Gesellschaft eine angemessene Kommunikation zwischen den betroffenen Instanzen sichergestellt sein muss. Angesichts der durch «enge Gesetzestreue» entstandenen Hindernisse hatten wir damals nur die Möglichkeit informeller persönlicher Kontakte. Wenn wir also unsere Verantwortung wahrnehmen wollten, mussten w ir davon Gebrauch machen. Auf diese Weise liess sich das Wesentliche schützen. Dies hing jedoch vom Vertrauensverhältnis zu unseren Kollegen ab. Vergleichbare Fragen stellen sich auch innerhalb des Kantons - zwischen der Standeskommission der Ärztegesellschaft und dem Kantonsarzt. In guter Gesellschaft wurde das Bedürfnis verstanden, der Aufsichtsbehörde schwerwiegende Elemente bezüglich einer Fachperson mitzuteilen. Dies war damals jedoch nicht formell geregelt. Wie ich bereits des Öfteren ausführte, ist ein Neben- einander von privaten und öffentlichen disziplinarischen Verfahren adäquat und praktisch. Die Privaten kümmern sich rascher um Streitfälle, die Mitglieder der Verbindung untereinander betreffen. Im Übrigen zeigt die Erfahrung, dass diese objektiv gesehen nicht alle gravierend sind. Wenn die Verbandskommission hingegen von schwerwiegenden Verfehlungen, Kunstfehlern oder sehr unangemessenen Verhaltensweisen (unter anderem mit sexuellem Bezug) hört, sind die Modalitäten zur Kommunikation mit der kantonalen Behörde festzulegen. Diesbezüglich sind die derzeitigen Schritte zu begrüssen, die im Rahmen der FMH unternommen werden. Bleibt bloss zu hoffen, dass die Umsetzung nicht auf halbem Wege stehen bleibt. Wie Cueni et al. feststellen, schöpfen «die Standeskommissionen ihre Sanktionsmöglichkeiten sehr oft zu wenig» aus.

Die Autoren weisen mit Recht auf dieses verwaltungsrechtliche Element hin, das in der deontologischen Praxis des Verbandes analog angewandt wurde. Die Klägerin wird dabei nicht als Partei betrachtet und hatte somit keinen Zugang zum Inhalt der Beweisaufnahme. Darüber hinaus wurde sie nach deren Ablauf lediglich darüber informiert, dass ein Beschluss gefasst wurde, ohne aber dessen Inhalt zu kennen. Dies stellt eine anachronistische und vor allem unausgewogene Situation dar (um einen Parteistatus zu erwerben, muss ein Strafverfahren mit den damit verbundenen Kosten und Fristen angestrebt werden). Ein Dankeschön an die Ärztekammer der FMH, die diese Situation mit ihrem Entscheid von 2013 korrigierte.

Die von Cueni et al. beschriebenen Ausrichtungen sind notwendig und angebracht. Dies trifft auch auf den Grundsatz eines Austauschs zwischen den Kommissionen der kantonalen Gesellschaften, der Arbeitstage und einer nationalen Datenbank zu.

Noch ein Gedanke: Damals stellte sich die Frage, ob sich eine beklagte Fachperson hinter dem Arztgeheimnis «verstecken» könnte. In der Folge wurde eine Verfügung (Art. 80a) in das Waadtländer Gesundheitsgesetz aufgenommen, die besagt, dass sich eine solche Person «nicht unter Berufung auf das Berufsgeheimnis weigern kann, die Gesundheitsbehörden über Fakten zu informieren, die zur Anklage führten, und dass sie sich ebenso wenig weigern kann, bei Konflikten zwischen ihr und ihren Patienten auf ziviler Ebene eine Aussage zu machen». 